\title{
Successful Treatment of Allergic Bronchopulmonary Aspergillosis Using a Combination of Inhaled Fluticasone Furoate/Vilanterol and Oral Voriconazole
}

\author{
Kanako Nishimatsu a, Seigo Minami ${ }^{\mathrm{a}, \mathrm{c}}$, Shoko Ikuta ${ }^{\mathrm{a}}$, \\ Shoichi Ihara ${ }^{\text {a, }}$, Kiyoshi Komuta ${ }^{\text {a, b }}$
}

\begin{abstract}
Systemic corticosteroids are considered to be the standard treatment for allergic bronchopulmonary aspergillosis (ABPA). However, there is controversy regarding use of inhaled corticosteroid (ICS) therapy for ABPA. Here we report a case of ABPA that was successfully treated with inhaled fluticasone furoate/vilanterol (FF/VI) and oral voriconazole (VRCZ). The patient was a 62-year-old Japanese man with bronchiectasis and diabetes mellitus who presented with fever, cough, and purulent sputum. Computed tomography scans of the chest showed consolidation in the left upper and lower lobes. Laboratory investigations revealed an abnormal increase in the number of eosinophils $\left(3,340 / \mathrm{mm}^{3}\right)$ and elevated levels of Creactive protein $(3.04 \mathrm{mg} / \mathrm{dL})$ and serum immunoglobulin $\mathrm{E}(\mathrm{IgE})$ $(763 \mathrm{U} / \mathrm{mL})$. Eight days after admission, he experienced a sudden attack of asthma. Aspergillus-precipitating antibodies were positive and Aspergillus fumigatus was detected in sputum culture. These results were consistent with a diagnosis of ABPA, and he was started on inhaled FF/VI and oral VRCZ. Systemic corticosteroids were not used because of the patient's history of diabetes mellitus and left atrial thrombus. His symptoms and consolidation improved significantly after treatment. He has not experienced an exacerbation for more than 3 years. In mild cases of ABPA in which total $\operatorname{IgE}$ is relatively low, inhaled FF/VI in combination with oral VRCZ can be considered as an alternative treatment to systemic corticosteroids in patients with ABPA.
\end{abstract}

Keywords: Allergic bronchopulmonary aspergillosis; Inhaled corticosteroids; Fluticasone furoate/vilanterol; Voriconazole; Itraconazole; Bronchial asthma; Aspergillus fumigatus; Bronchiectasis

Manuscript submitted August 18, 2020, accepted August 29, 2020

Published online September 23, 2020

aDepartment of Respiratory Medicine, Osaka Police Hospital, 10-31 Kitayama-cho, Tennoji-ku, Osaka 543-0035, Japan

bepartment of Respiratory Medicine, Daini Osaka Police Hospital, 2-6-40 Karasuga-tuji, Tennoji-ku, Osaka 543-0042, Japan

${ }^{\mathrm{c} C}$ Corresponding Author: Seigo Minami, Department of Respiratory Medicine, Osaka Police Hospital,10-31 Kitayama-cho, Tennoji-ku, Osaka 543-0035, Japan. Email: seigominami@oph.gr.jp

doi: https://doi.org/10.14740/jmc3567

\section{Introduction}

Allergic bronchopulmonary aspergillosis (ABPA) is a disease in which there is an allergic reaction to Aspergillus, which damages the airways, leading to structural changes in the bronchus, obstruction, and asthma.

Systemic steroid therapy is generally the first choice for ABPA [1]. However, inhaled corticosteroid (ICS) therapy can be considered as an adjuvant therapy to reduce the dose of systemic steroids or to reduce the severity of bronchial asthma [2, 3]. There have been some reports of patients in whom addition of ICS successfully reduced the dose of systemic steroids required $[4,5]$ and of ICS monotherapy providing symptomatic relief in patients who refused treatment with oral steroids [6]. In a retrospective study performed in India [2], ICS as monotherapy-controlled symptoms but failed to suppress serum IgE levels, which are a useful indicator of ABPA disease activity [3]. Therefore, use of ICS therapy in ABPA remains controversial.

Itraconazole (ITCZ) is an antifungal agent with the most evidence for efficacy in ABPA. Voriconazole (VRCZ), posaconazole, and inhaled amphotericin $\mathrm{B}$ are alternative antifungal choices when ITCZ is ineffective or cannot be tolerated [7]. Both ITCZ and VRCZ are available in oral formulations and have high antifungal activity against Aspergillus and trivial side effects. Therefore, these drugs are suitable for long-term treatment in an outpatient setting. VRCZ may be a more effective treatment for ABPA than ITCZ because of better activity against Aspergillus fumigatus, a lower incidence of resistance, and better bioavailability [8].

Here we report a case of ABPA that responded well to a combination of inhaled ICS and oral VRCZ.

\section{Case Report}

A 62-year-old Japanese man presented with a fever of 38.0 ${ }^{\circ} \mathrm{C}$, cough, and purulent sputum. He was a former smoker of 15 cigarettes per day for 38 years. A chest radiograph showed consolidation in the left upper lung field. We suspected bacterial pneumonia and treated him with clavulanic acid $500 \mathrm{mg}$ /day and amoxicillin $2000 \mathrm{mg} /$ day in combina- 


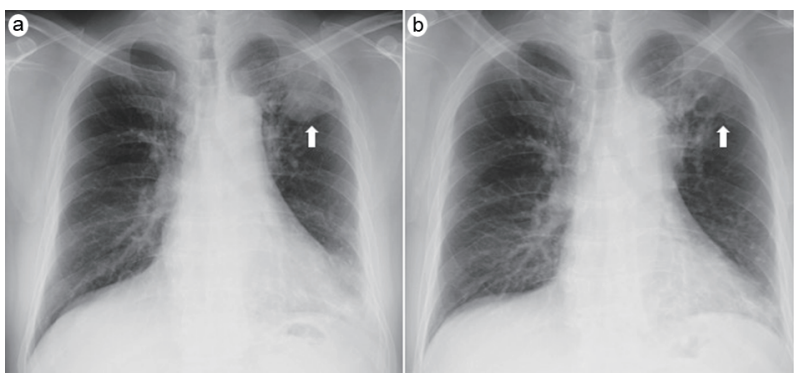

Figure 1. Chest radiographs on admission (a) and on day 12 in hospital (b). The consolidation in the left upper lung field (arrow) on admission had improved by day 12 .

tion with azithromycin $2000 \mathrm{mg} /$ day. Seven days later, he was admitted when chest radiography indicated exacerbation of the consolidation (Fig. 1a) and a computed tomography scan of the chest suggested consolidation with mucus plugs and cystic bronchodilatation in the left upper and lower lobes (Fig. 2a).

He had visited our department regularly for bronchiectasis for 7 years. His past medical history also included hypertension, diabetes mellitus, chronic kidney disease, myocardial infarction, left atrial thrombus, and bacterial pneumonia. However, he had no history of bronchial asthma. His regular medications included clopidogrel, rabeprazole, olmesartan, pitavastatin, azosemide, carvedilol, warfarin, metformin, saxagliptin, mosapride, and trimebutine. Seven years earlier, eosinophilia had been detected in peripheral blood samples and Aspergillus was detected in sputum. These findings did not meet the criteria for ABPA at that time. He was treated with systemic steroids for only 2 months. Thereafter, eosinophilia was not observed until the present event. His height, weight, body temperature, blood pressure, heart rate, and percutaneous oxygen saturation (in ambient air) on admission were $157.5 \mathrm{~cm}, 64.8 \mathrm{~kg}, 35.7^{\circ} \mathrm{C}$, $113 / 78 \mathrm{~mm} \mathrm{Hg}, 88$ beats $/ \mathrm{min}$, and $97 \%$, respectively. His lung sounds were clear. The leukocyte count was 16,700 / $\mathrm{mm}^{3}$, and eosinophils accounted for $20 \%$ of the leukocytes. Serum C-reactive protein and immunoglobulin E (IgE) levels were elevated to $3.04 \mathrm{mg} / \mathrm{dL}$ and $763 \mathrm{U} / \mathrm{mL}$, respectively. Although $\beta$-D-glucan and Aspergillus antigen were both negative, anti-Aspergillus $\mathrm{IgG}$ antibody and serum A. fumigatus-specific IgE antibody were both positive. On admission, we initially suspected bacterial pneumonia and administered intravenous tazobactam/piperacillin $4.5 \mathrm{~g}$ four times daily. However, 8 days after admission, he developed wheeze. No reversibility was observed on an airway reversibility test. Compared with the spirometry results from 1 year earlier, there was a decrease in forced expiratory volume in $1 \mathrm{~s}\left(\mathrm{FEV}_{1}\right)$ from 1.47 to $1.28 \mathrm{~L}$ and in forced maximum expiratory flow from 5.22 to $3.57 \mathrm{~L} / \mathrm{s}$. The percent predicted $\mathrm{FEV}_{1}\left(\% \mathrm{FEV}_{1}\right)$ was $79.0 \%$. The exhaled nitric oxide level was $123 \mathrm{ppb}$. We diagnosed bronchial asthma and started inhaled fluticasone furoate $200 \mu \mathrm{g}$ and vilanterol $25 \mu \mathrm{g}$ (FF/ VI) therapy. Tazobactam/piperacillin was considered ineffective and discontinued. On day 12 of his hospital stay (5 days after introduction of FF/VI), the consolidation on his

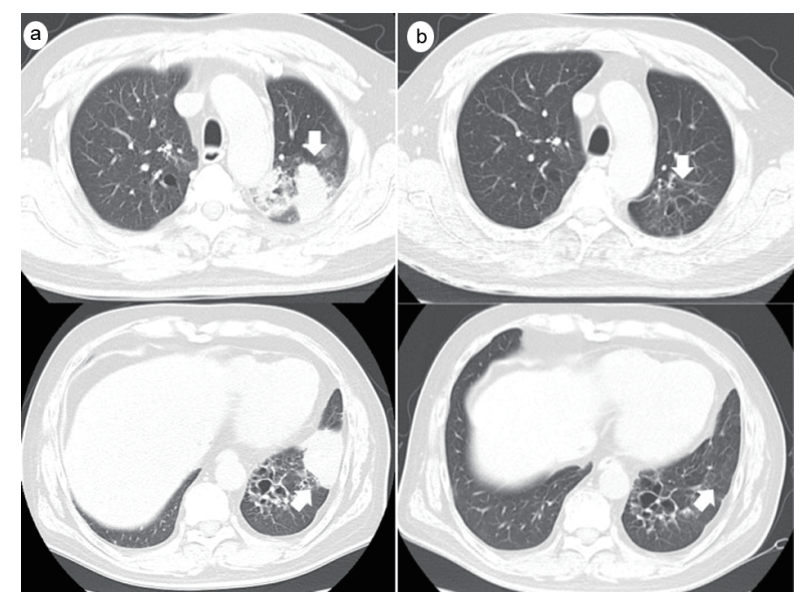

Figure 2. Computed tomography scans on admission (a) and 2 months after discharge (b). The consolidation, mucus plugs, and cystic bronchodilatation seen in the left upper and lower lobes (arrows) on admission had disappeared 2 months later.

chest radiograph was improved (Fig. 1b) and his wheezing had disappeared. On day 13, we performed bronchoscopy. Bronchoalveolar lavage of the left upper lobe yielded a fluid recovery rate of $36.7 \%(55 / 150 \mathrm{~mL})$ with $3.9 \times 10^{6}$ nucleated cells $/ \mathrm{mL}, 22.5 \%$ eosinophils, $17.5 \%$ lymphocytes, and $56.5 \%$ macrophages. Mucus plugs were found in the left $\mathrm{B}^{1+2 b}$. No Grocott's methenamine silver stained bacteria or other significant organisms were found in the bronchoalveolar lavage fluid. No fungus was detected by Periodic acidSchiff or Grocott's methenamine silver stain in the transbronchial lung biopsy, with only inflammatory cells, such as lymphocytes, neutrophils, and macrophages, infiltrating into the alveoli. A. fumigatus was detected in a sputum culture obtained on day 8. Although the serum total $\operatorname{IgE}$ level was less than $1,000 \mathrm{U} / \mathrm{mL}$, the findings met the International Society for Human and Animal Mycology diagnostic criteria for ABPA. We started VRCZ at a dose of $600 \mathrm{mg} /$ day followed by a maintenance dose of $400 \mathrm{mg} /$ day. We did not start systemic steroid therapy in view of his diabetes mellitus and history of left atrial thrombus. The combination of oral VRCZ and inhaled FF/VI rapidly improved his symptoms, consolidation on chest radiography (Fig. 1b), eosinophilia, and elevated C-reactive protein levels. He was discharged on day 27 . We were able to maintain his trough blood VRCZ concentration within the range of $3.9-4.9 \mu \mathrm{g} /$ $\mathrm{mL}$ and simultaneously adjusted his warfarin dose according to the prothrombin time-international normalized ratio (PT-INR). By 2 months after discharge, the consolidation had disappeared on a chest computed tomography scan (Fig. $2 \mathrm{~b}$ ), and $\mathrm{FEV}_{1}$ and forced maximum expiratory flow recovered to $1.56 \mathrm{~L}$ and $5.54 \mathrm{~L} / \mathrm{s}$, respectively.

Six months later, VRCZ was discontinued because there had been no exacerbations or difficulties in controlling his PTINR due to interaction with warfarin. At that time, the serum IgE level was still $725 \mathrm{U} / \mathrm{mL}$ but he remained asymptomatic while on FF/VI therapy alone. Thereafter, the serum IgE level remained in the range of $700-1000 \mathrm{U} / \mathrm{mL}$. The exhaled nitric oxide level had decreased to $22 \mathrm{ppb}$ by 1 year after discharge. 
No exacerbation has occurred in the 3 years since that admission.

\section{Discussion}

This case is interesting in that we could successfully control ABPA using only inhaled FF/VI therapy and VRCZ without systemic steroid therapy and indicates that some cases of ABPA can be treated with ICS and an anti-fungal agent. In a single-center, non-blinded randomized trial from India that compared systemic steroid therapy with VRCZ in acute-phase ABPA with elevated total $\mathrm{IgE}>1000 \mathrm{IU} / \mathrm{mL}$ [9], there was no difference in the effect of treatment on time to first exacerbation or adverse events. In that trial, treatment with an inhaled ICS, formoterol (a long-acting $\beta_{2}$-agonist (LABA)), and montelukast was allowed in both study groups. All patients received ICS/LABA and 44\% received montelukast, respectively. Therefore, combination therapy with antifungal drugs and inhaled ICS/LABA may be similar in effect to systemic steroid therapy in ABPA. Total IgE was elevated to $>1,000$ $\mathrm{IU} / \mathrm{mL}$ in all patients in retrospective studies from India and the US that concluded there is no role for ICS monotherapy in the management of ABPA [2, 3]. In contrast, in a case report that included two patients and suggested that ICS therapy is useful in ABPA [5], the peak total IgE was $414 \mathrm{IU} / \mathrm{mL}$ in one patient and $214 \mathrm{IU} / \mathrm{mL}$ in the other. It is possible that patients, in whom the total IgE level is not very high, suggesting a local allergic reaction, can be treated by ICS alone.

Our case suggests that $\mathrm{VRCZ}$ is a promising alternative antifungal drug to ITCZ in patients with ABPA. In an Indian study that compared systemic steroid therapy with ITCZ for acute ABPA [10], ICTZ was inferior in terms of the composite response after 6 weeks of systemic steroid therapy but was effective in $88 \%$ of cases. However, in another trial from India that compared systemic steroid therapy with $\mathrm{VRCZ}$ in acute-phase ABPA, VRCZ was very similar in composite response to prednisolone after 6 weeks $(96 \%)$ [9]. Furthermore, the US Food and Drug Administration Adverse Event Reporting System suggested that ITCZ should not be administered in patients with evidence of ventricular dysfunction, such as congestive heart failure or a history of congestive heart failure [11]. Therefore, we selected VRCZ in this case.

In conclusion, inhaled FF/VI in combination with oral VRCZ can be considered as an alternative to systemic corticosteroid therapy in mild cases of ABPA where total $\operatorname{IgE}$ is relatively low.

\section{Acknowledgments}

The authors thank Dr. Hironao Yasuoka, Department of Pathology, Osaka Police Hospital, for his guidance on the pathological findings in this case.

\section{Financial Disclosure}

None to declare.

\section{Conflict of Interest}

None to declare.

\section{Informed Consent}

The patient consented to inclusion of his clinical information in this report.

\section{Author Contributions}

KN contributed mainly to the patient's treatment. KN and SM drafted the manuscript. All authors contributed to patient care and read and approved the final manuscript.

\section{Data Availability}

The authors declare that the data supporting the findings of this case are available within the article.

\section{References}

1. Patterson TF, Thompson GR, 3rd, Denning DW, Fishman JA, Hadley S, Herbrecht R, Kontoyiannis DP, et al. Practice guidelines for the diagnosis and management of aspergillosis: 2016 update by the Infectious Diseases Society of America. Clin Infect Dis. 2016;63(4):e1-e60.

2. Agarwal R, Khan A, Aggarwal AN, Saikia B, Gupta D, Chakrabarti A. Role of inhaled corticosteroids in the management of serological allergic bronchopulmonary aspergillosis (ABPA). Intern Med. 2011;50(8):855-860.

3. Greenberger PA, Bush RK, Demain JG, Luong A, Slavin RG, Knutsen AP. Allergic bronchopulmonary aspergillosis. J Allergy Clin Immunol Pract. 2014;2(6):703-708.

4. Machida K, Tsuda T, Osaki S, Inoue H, Tsuda M, Kido M. [A case of allergic bronchopulmonary aspergillosis followed by diagnostic imaging for 27 years]. Nihon Kokyuki Gakkai Zasshi. 2005;43(6):379-383.

5. Imbeault B, Cormier Y. Usefulness of inhaled high-dose corticosteroids in allergic bronchopulmonary aspergillosis. Chest. 1993;103(5):1614-1617.

6. Balter MS, Rebuck AS. Treatment of allergic bronchopulmonary aspergillosis with inhaled corticosteroids. Respir Med. 1992;86(5):441-442.

7. Tracy MC, Okorie CUA, Foley EA, Moss RB. Allergic bronchopulmonary aspergillosis. J Fungi (Basel). 2016;2(2).

8. Johnson LB, Kauffman CA. Voriconazole: a new triazole antifungal agent. Clin Infect Dis. 2003;36(5):630-637.

9. Agarwal R, Dhooria S, Sehgal IS, Aggarwal AN, Garg M, Saikia B, Chakrabarti A. A randomised trial of voriconazole and prednisolone monotherapy in acute-stage allergic bronchopulmonary aspergillosis complicating asthma. Eur Respir J. 2018;52(3). 
10. Agarwal R, Dhooria S, Singh Sehgal I, Aggarwal AN, Garg M, Saikia B, Behera D, et al. A randomized trial of itraconazole vs prednisolone in acute-stage allergic bronchopulmonary aspergillosis complicating asthma. Chest.
2018;153(3):656-664.

11. Ahmad SR, Singer SJ, Leissa BG. Congestive heart failure associated with itraconazole. Lancet. 2001;357(9270):17661767. 